\title{
The capricious relationship between recognition and outcome of mental illness in primary care
}

\author{
BEA G. TIEMENS AND JOHAN ORMEL
}

Recognition of mental disorders by the General Practitioner (GP) often has been described as insufficient. Factors causing non recognition vary from patients characteristics and problems to skills and attitudes of the GPs. To make GPs more sensitive to psychological disorders post-graduate training programs and guidelines have been developed. Some of these programs have been proved to be effective in increasing recognition, but to date evidence that improved detection substantially improves prognosis has been scarce. Different studies, performed over a period of 20 years and summarized in table I, showed inconsistent results. A first quick sight at this table suggests that recognition does not make a difference; two third of the studies showed no association between recognition and better outcome. In this editorial we elaborate why studies may frequently fail to find an association between recognition and better outcome. To this end, we make a distinction between methodological factors, factors related to the design or the sample of the study, and factors regarding the clinical consequences of recognition. We will argue that a methodological as well as a clinical factor are critically important for recognition to improve outcome, being whether the study sample included incident or prevalent cases and whether or not recognition is followed by adequate treatment.

\section{WHAT FACTORS CAN ACCOUNT FOR NOT FINDING AN ASSOCIATION BETWEEN RECOGNITION AND BETTER OUTCOME?}

\section{Study design}

In four of the six studies which did not find an

Indirizzo per la corrispondenza: Prof. B.G. Tiemens, Department of Psychiatry, University of Groningen, PO Box 30.001, 9700 RB Groningen, The Netherlands.

Fax $+31-50-369.6727$. association between recognition and better outcome the observational study design could be the cause of this. Among the five observational studies only the study of Ormel et al. (1990) showed an association between recognition and better outcome. Because of the observational nature of these studies, it can not be excluded that the outcome of recognized cases would have been worse if they had not been recognized. In this instance, recognition does make a difference, but the observational design is not capable to detect differences in outcomes between recognized and unrecognized cases. This is supported by the finding that in most studies recognized patients differ from the unrecognized. Recognized patients tend to have more severe psychopathology and more impairment in occupational functioning, what makes their prognosis worse (Ormel et al., 1993; Katon, 1995). Reckoning with this point by controlling for initial severity, however, did not change the result of our most recent study that outcome was unrelated to recognition (Tiemens et al., 1996).

From the experimental studies, those of Johnstone \& Goldberg (1976) and of Zung et al. (1983) showed positive results of feedback on outcome in contrast to the studies of Mathias et al. (1994) and of Dowrick \& Buchan (1995). In these experimental studies, the lack of an effect of recognition on outcome might be due to the well-known phenomenon of the high rate of false positives in a population with low prevalence of the condition for which we screen (Samuelson, 1995). In the experimental studies, feedback about the presence of psychological problems in screen-positive patients was provided to the GPs on a randomized basis. The phenomenon Samuelson described, assumes high rates of false positives among screen-positive patients who were not detected by the GP; the patients with hidden psychiatric morbidity. However, with prevalence rates varying from $10 \%$ to $25 \%$ in most studies of primary care attenders, prevalence of psychological disorders can not be considered low. Moreover, in the 
Table I. - Recognition and outcome studies.

\begin{tabular}{|c|c|c|c|c|c|}
\hline Study & $\begin{array}{l}\text { Observational/ } \\
\text { Experimental }\end{array}$ & $\begin{array}{l}\text { Incident/ } \\
\text { Prevalent } \\
\text { cases }\end{array}$ & $\begin{array}{l}\text { Recognition always } \\
\text { included treatment? }\end{array}$ & Study period & $\begin{array}{l}\text { Association beetwen } \\
\text { recognition and } \\
\text { better outcome? }\end{array}$ \\
\hline Johnstone \& Goldberg, 1976 & Experimental & Prevalent & Yes & 1 year & Yes \\
\hline Zung et al., 1983 & Experimental & Incident & Yes & 4 weeks & Yes \\
\hline Schulberg et al., 1987 & Observational & Prevalent & No & 6 months & No \\
\hline Ormel et al., 1990 & Observational & Incident & No & 1 year & Yes \\
\hline Coyne et al., 1994 & Observational & Prevalent & No & 9 months & No \\
\hline Matthias et al., 1994 & Experimental & Prevalent & No & 5 months & No \\
\hline Dowrick \& Buchan, 1995 & Experimental & Prevalent & No & 1 year & No \\
\hline Simon \& Von Korff, 1995 & Observational & Prevalent & No & 1 year & No \\
\hline Tiemens et al., 1996 & Observational & Prevalent & No & 1 year & No \\
\hline
\end{tabular}

study of Johnstone and Goldberg, feedback after screening had the same positive effect on outcome (of hidden psychiatric morbidity) as detection by the GP (on "conspicuous psychiatric morbidity»).

\section{Study sample}

Table I shows that two of the three studies that found an association between recognition and better outcome, included only incident cases. The inclusion of prevalent cases in the observational studies could have diluted the effect of recognition, because prevalent cases can have been recognized on earlier visits and have been unresponsive to treatment. Patients who remained ill despite recognition (and possibly treatment) are less likely to improve. Consistent with this hypothesis is that the study of Ormel et al. (1990) which found an association between recognition and better outcome excluded prevalent cases.

In the experimental studies the inclusion of prevalent cases might account for not finding an effect of recognition too, but for other reasons than in the observational studies. First, because prevalent cases include a relatively high proportion of high utilizers of care who have a higher than average probability of multiple physical or social problems, which may have diluted the effect of recognition on outcome (Dowrick \& Buchan, 1995). The second reason emphasizes the longer delay in the delivery of treatment amongst prevalent «feedback» patients as compared to incident «feedback» patients. Scott et al. (1992) found that the length of the interval between onset of depression and treatment predicted prognosis; the shorter the no-treatment interval, the better the prognosis. The patients about whom the GPs received feedback in the experimental studies, had not been recognized and treated during earlier visits. The delay could have worsened their prognosis. However, the "delay hypothesis» is not supported by the study of Johnstone \& Goldberg (1976), who found that recognition of prevalent cases with severe disorders was associated with both a short-term effect (at 3, 6 and 9 months) and a longterm effect (at 12 months).

\section{Clinical factors}

An important factor in observational as well as experimental studies is that recognition does not necessarily imply adequate treatment, i.e. treatment according to clinical guidelines. Feedback about the mental health status of the patient may only be consequential when the GP has received adequate training in how to use this feedback effectively. The studies of Johnstone \& Goldberg (1976) and Zung (1983), provide circumstantial evidence in favour of this hypothesis. Both studies found that recognition followed by treatment resulted in better outcomes. However, to date the number of randomized treatment trials in primary care has been small, and it also can not be excluded that the efficacy of treatments found in randomized clinical trials with psychiatric (out)patients will not hold up for primary care patients. Transfer of efficacy rates obtained in psychiatric specialty settings to effectiveness estimates for primary care settings requires that three conditions are met: a) similarity of the treatment setting, (b) qualitative similarity of the disorder, i.e. similar aetiologies, and (c) similar patient characteristics. (Ormel \& Tiemens, 1995). Similarity of treatment settings is moderate to low, due to differences in the duration of visits and in the reimbursement 
schedules. Whether disorders are qualitatively similar in both settings is difficult to assess. Obviously, the case-mix is different, with the more severe cases being relatively more prevalent in specialty settings. However, this does not imply qualitative differences. There is a difference in the long-term course, with specialty patients showing more often persistence of symptoms, relapses and recurrences than primary care patients (Cooper-Patrick et al., 1994). This may be indicative of low qualitative similarity, in as far as the difference in course indicates different aetiologies, in terms of vulnerability and illnessmaintaining factors. The third assumption of similar patient characteristics is definitely not met. The typical randomized-clinical-trial-patient in psychiatry is better motivated, with a well-defined psychiatric disorder, not too old and without serious medical comorbidity. The typical distressed primary care patient is different, with more often milder but mixed anxiety and depressive symptoms, more often physical comorbidity, more often reluctance to accept a psychiatric diagnosis and less motivation to take medicines. Transfer of treatment response rates from psychiatric specialty into primary care settings is therefore problematic.

Nevertheless, consensus about management of psychological disorders in primary care is developing. Compared to these clinical guidelines, current treatment in primary care is often inadequate. Furthermore, evidence is growing that treatment according to these guidelines, i.e. dosage and duration of anti-depressant medication is adequate and accompanied with psycho-education, improves patient adherence and outcome (Lin et al., 1995; Katon et al., 1995). Positive results have also been reported for non-pharmacological interventions in primary care (Catalan et al., 1991, Mynors-Wallis et al., 1995).

\section{HOW TO IMPROVE OUTCOME?}

The "capricious» relationship between recognition and outcome suggests that we shift focus and efforts from improving recognition to improving outcome. It must be clear that improving recognition alone is a necessary, but by no way a sufficient condition for better outcome. To improve outcome, we emphasize four points of actions:

1. First it is important to improve early detection of mental illness. Early detection in primary care is difficult because most patients visit their doctor with a somatic complaint. Especially patients who only express their somatic symptoms to their doctor benefit from recognition (Ormel et al., 1990). Ormel's findings suggest that recognition may also improve outcome through other paths than pharmaco- or psychotherapy. They mentioned the following healing effects of recognition: acknowledgement of patient's distress, reinterpretation of somatic complaints within a stress framework, and social support.

2. Second, GPs need to provide adequate, evidence-based treatment, that is treatment that has been proved to be effective in randomized clinical trials in primary care.

The best strategy for achieving the objectives 1 and 2, is to develop programs that train GPs in diagnostic and treatment protocols, and provide practical tools that help them in the use of these protocols. There is some evidence that training of general practitioners in detection, diagnostics and management of psychological disorders, improves (temporarily) the process of care and patient outcomes, including reduction of suicide rates (Roter et al., 1995; Rutz et al., 1989).

3. The third point of action is that we need more and better designed clinical trials, which evaluate training programs for GPs and treatment modalities. To date, too little is known about what type of patients benefit from what type of treatments. In future trials response prediction should become an essential component in order to improve the specificity of indication criteria for various practical treatment modalities.

4. Finally, the focus should not be exclusively on acute treatment, but also on long-term management. There is evidence now that depression has a recurrent or chronic course in at least half of the patients with a first episode. Considering depression as a chronic-recurrent illness requires supplementing acute pharmacological or psychotherapeutic treatment with long-term management. Programs for enhancement of self-management developed along the lines of those for somatic chronic diseases may also be helpful for patients with mental disorders (Von Korff et al., 1996). These programs are based on the principles of social learning, i.e. constructive thinking, planning of pleasant activities, relaxation techniques, and social skills (as in the «Coping with Depression Course» of Lewinsohn et al., 1984), and of self-regulation i.e. self-monitoring of symptoms and relapse prevention strategies. Development of 
programs suitable for primary health care settings and testing those on effectiveness in prevention of relapse/recurrence, can be an important step in improving outcome.

In summary, our hypothesis is that outcome can be improved by early detection especially if it is followed by evidence-based acute treatment and additional self-management enhancement programs. Therefore future efforts should not be aimed at resolving the "recognition and outcome mystery», but at the development and testing of training programs for GPs and treatment modalities for patients in primary care settings.

\section{REFERENCES}

Catalan J., Gath D.H., Anastasiades P., Bond S.A.K., Day A. \& Hall L. (1991). Evaluation of a brief psychological treatment for emotional disorders in primary care. Psychological Medicine 21, 1013-1018.

Cooper-Patrick L., Crum R.M. \& Ford D.E. (1994). Characteristics of patients with major depression who received care in general medical and specialty mental health settings. Medical Care 32, 15-24.

Coyne J.C. \& Schwenk T.L. (1994). Reconsidering the problem of undetected depression among primary medical care patients. Presented at the 8th Annual NIMH Int Res Conf on Mental health Problems in the General Health Care Sector, McLea, Virginia, September 7-9.

Dowrick C. \& Buchan I. (1995). Twelve month outcome of depression in general practice: does detection or disclosure make a difference? British Medical Journal 311, 1274-1276.

Johnstone A. \& Goldberg D. (1976). Psychiatric screening in general practice. A controlled trial. Lancet 20, 605-609.

Katon W. (1995). Will improving detection of depression in primary care lead te improved depressive outcomes? General Hospital Psychiatry 17, 1-2.

Katon W., Von Korff M., Lin E., Walker E., Simon G.E., Bush T., Robinson P. \& Russo J. (1995). Collaborative management to achieve treatment guidelines. Impact on depression in primary care. Journal of American Medical Association 273, 1026-1031.

Lewinsohn P.M., Antonuccio D.O., Breckenridge J.S. \& Teri L. (1984). The Coping with Depression Course. A Psychoeducational Intervention for Unipolar Depression. Castalia Publishing Company: Eugene.
Lin E.H.B., Von Korff M., Katon W., Bush T., Simon G.E., Walker E. \& Robinson P. (1995). The role of the primary care physician in patients adherence to antidepressant therapy. Medical Care 33, 67-74.

Mathias S.D., Fifer S.K., Mazonson P.D., Lubeck D.P., Buesching D.P. \& Patrick D.L. (1994). Necessary but not sufficient: the effect of screening and feedback on outcomes of primary care patients with untreated anxiety. Journal of General Internal Medicine 9, 606-615.

Mynors-Wallis L.M., Gath D.H., Lloyd-Thomas A.R. \& Tomlinson D. (1995). Randomised controlled trial comparing problem solving treatment with amitriptyline and placebo for marjor depression in primary care. British Medical Journal $310,441-445$.

Ormel J. \& Tiemens B. (1995). Recognition and treatment of mental illness in primary care. Towards a better understanding of a multifaceted problem. General Hospital Psychiatry 17, 160164.

Ormel J., Brink W. van den, Koeter M.W.J., Giel R., Meer K. van der, Willige G. van de \& Wilmink F.W. (1990). Recognition, management and outcome of psychological disorders in primary care: a naturalistic follow-up study. Psychological Medicine $20,909-923$.

Ormel J., Oldehinkel T., Brilman E. \& Brink W. van den (1993). Outcome of depression and anxiety in primary care. A threewave 3-year study of psychopathology and disability. $A r$ chives of General Psychiatry 50, 759-766.

Roter D.L., Hall J.A., Kern D.E., Barker R., Cole K.A. \& Roca R.P. (1995). Improving physicians interviewing skills and reducing patients'emotional distress. Archives of Internal Medicine $155,1877-1884$.

Rutz W., Knorring von L. \& Wílinder J. (1989). Frequency of suicide on Gotland after systematic postgraduate education of general practitioners. Acta Psychiatrica Scandinavica 80, 151154.

Samuelson P. (1995). Unrecognized mental illness in primary care. Archives of Family Medicine 4, 581.

Schulberg H.C., McClelland M. \& Gooding W. (1987). Six-month outcomes for medical patients with major depressive disorders. Journal of General Internal Medicine 2, 312-317.

Scott J., Eccleston D. \& Boys R. (1992). Can we predict the persistence of depression? British Journal of Psychiatry 161, 633-637.

Simon G.E. \& Von Korff M. (1995). Recognition, management, and outcomes of depression in primary care. Archives of $\mathrm{Fa}$ mily Medicine 4, 99-105.

Tiemens B.G., Ormel J. \& Simon G.E. (1996). Occurrence, recognition, and outcome of psychological disorders in primary care. American Journal of Psychiatry 153, 636-644.

Von Korff M., Gruman J., Schaefer J., Curry S. \& Wagner E.H. (1996). Self-management training and support: requirements for health care integration. Personal communication.

Zung W.W.K., Magill M., Moore J.T. \& George D.T. (1983). Recognition and treatment of depression in a family medicine practice. Journal of Clinical Psychiatry 44, 3-6. 